\title{
Evaluation of a multimode fiber optic low coherence interferometer for path length resolved Doppler measurements of diffuse light
}

\author{
Babu Varghese, ${ }^{\text {a) }}$ Vinayakrishnan Rajan, Ton G. Van Leeuwen, ${ }^{\text {b) }}$ and \\ Wiendelt Steenbergen \\ Institute for Biomedical Technology, Biophysical Engineering Group, University of Twente, P.O. Box 217, \\ NL-7500AE Enschede, The Netherlands
}

(Received 17 July 2007; accepted 19 November 2007; published online 26 December 2007)

\begin{abstract}
The performance of a graded index multimode fiber optic low coherence Mach-Zehnder interferometer with phase modulation is analyzed. Investigated aspects were its ability to measure path length distributions and to perform path length resolved Doppler measurements of multiple scattered photons in a turbid suspension of particles undergoing Brownian and translational motion. The path length resolution of this instrument is compared with a system using single mode fibers for illumination and detection. The optical path lengths are determined from the zero order moment of the phase modulation peak in the power spectrum. The weighted first moment, which is equal to the average Doppler shift, shows a linear response for different mean flow velocities within the physiological range. (c) 2007 American Institute of Physics. [DOI: 10.1063/1.2823558]
\end{abstract}

Path length resolved temporal fluctuations of photon intensity and the corresponding Doppler shift in dynamic media can be measured using low coherence interferometry. ${ }^{1-5}$ Path length distributions in static and mixed static-dynamic media can be measured by phase modulated low coherence Mach-Zehnder interferometer. ${ }^{6}$ This will enable path length resolved measurements in mixed media such as tissue perfused with blood. ${ }^{7}$ Since the use of single mode fibers does lead to a too low signal level in a dual fiber geometry as used in laser Doppler techniques, we take the advantage of multimode fibers to collect sufficient scattered light at large fiber distances. The aim of the present work is to establish the performance of the system in terms of its resolution of optical path length assessment and its ability to represent various flow speeds within the physiological velocity range. In previous reports, ${ }^{1-6}$ coherence gated optical Doppler measurements are performed on nonflowing samples, in which the Doppler shift was imparted due to Brownian motion of particles.

A broadband superluminescent diode (Inject LM2-850, $\lambda=832 \mathrm{~nm}, \Delta \lambda_{\mathrm{FWHM}}=17 \mathrm{~nm}$, coherence length $\left.L_{C}=18 \mu \mathrm{m}\right)$ that yields $2 \mathrm{~mW}$ of power from the single mode pigtail fiber is used as the light source. In contrast to single mode fiber optic interferometers used for path length resolved measurements, ${ }^{1-3}$ adopting on axis back reflection and confocal detection of singly scattered photons, our system uses multimode graded index fibers [core diameter $=100 \mu \mathrm{m}$, numerical aperture $(\mathrm{NA})=0.29$, length $=1 \mathrm{~m}]$ for detection of multiply scattered light, with positions for illumination (single mode fibers, mode field diameter $=5.3 \mu \mathrm{m}$, NA $=0.14$ ) and detection separated by a distance of $300 \mu \mathrm{m}$. This spatial separation corresponds to five times the scattering mean-free-path length in an aqueous suspension of $10 \%$

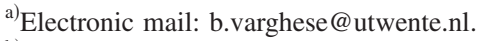

b) Also at Academic Medical Center, Laser Center, Meibergdreef 9, 1105 AZ, Amsterdam, The Netherlands.
}

of Intralipid $20\left(\mu_{\mathrm{s}}^{\prime}=5.0 \mathrm{~mm}^{-1}, \mu_{a}=0.001 \mathrm{~mm}^{-1}, g=0.637\right)$ that we used in this study. For measuring the path length resolution of single mode fiber optic interferometer, single mode fibers are used for detection. The coherence length of the light source, and the intermodal dispersion in the detection fiber, define the path length resolution of the measurement. The reference beam is polarized using a linear polarizer and the phase is sinusoidally modulated at $17 \mathrm{kHz}$ with a peak optical phase shift of less than $\pi / 2$ radians using an electro-optic broadband phase modulator (New Focus model 4002). The power spectra were measured as a function of the optical path length in the reference arm in steps of $20 \mu \mathrm{m}$ at positions where the optical path length difference is within the coherence length and in steps of $100 \mu \mathrm{m}$ for widely different optical path lengths.

For the velocity response test, a scattering model was made of Delrin with a circular straight flow channel (diameter of $3.8 \mathrm{~mm}$, length $=2.5 \mathrm{~cm}$ ) at a depth of $0.2 \mathrm{~mm}$ under the surface of the Delrin. An aqueous suspension of $10 \%$ of Intralipid $20 \%$ was used as a scattering medium $\left(\mu_{\mathrm{s}}^{\prime}\right.$ $\left.=5.0 \mathrm{~mm}^{-1}, \mu_{a}=0.001 \mathrm{~mm}^{-1}, g=0.637\right)$ and the mean flow through the channel was varied over a range of $0-6 \mathrm{~mm} / \mathrm{s}$ with a peristaltic pump. At the detector (New Focus model 2001 photoreceiver), the light that is scattered in the sample and the light from the reference arm are mixed. The ac photocurrent is measured with a 12 bit analog to digital converter (National Instruments), sampling at $40 \mathrm{kHz}$ for $58 \mathrm{~s}$ to get an average of 1000 spectra and were measured in steps of $100 \mu \mathrm{m}$.

In laser Doppler perfusion measurements, the flow parameters are obtained from the moments of power spectrum. ${ }^{8}$ The method presented here utilizes the area $M_{0}$ (zero order moment) and the weighted first moment $M_{1} / M_{0}$ of the phase modulation peak (after noise correction in a bandwidth of $14-20 \mathrm{kHz}$ ) appearing in the photodetector signal power spectrum to determine the intensity and the Doppler shift of photons as a function of optical path length, respectively. 


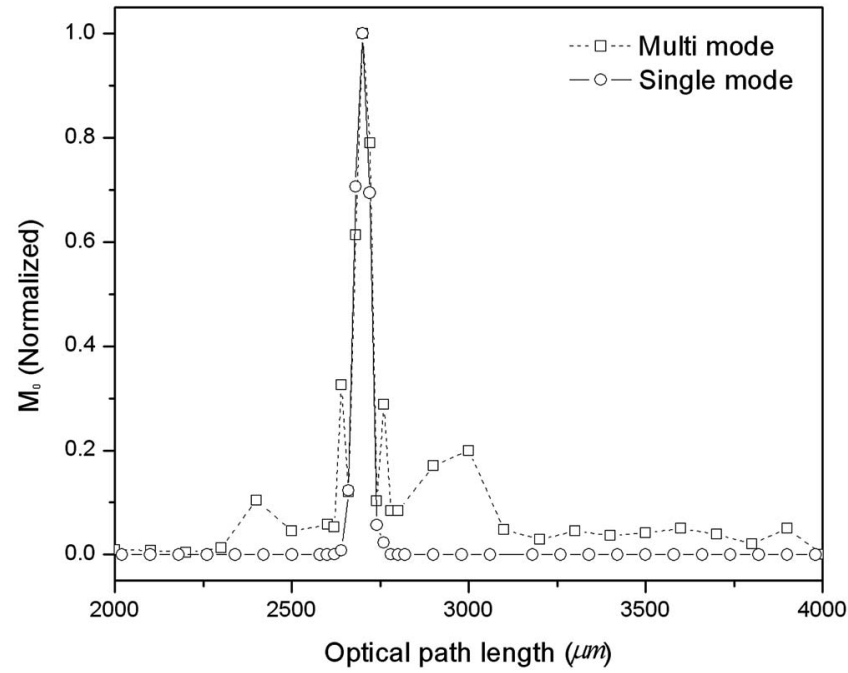

FIG. 1. Normalized total power, $M_{0} N$, of detector current $\left(M_{0}=\right.$ zeroth moment of power spectral density) as a function of the optical path length in the reference arm measured with multimode and single mode fiber optic interferometer.

To measure the path length resolution of the setup, the scattering sample was replaced by a reflecting mirror at a distance of $1.35 \mathrm{~mm}$ from the fiber tips. When the optical path length in the reference arm was about $2.7 \mathrm{~mm}$, we observed a narrow and strong interference peak at the modulation frequency due to the interference of reference light with the light reflected from the mirror. This is expected since the light has to pass twice, and this optical path length difference corresponds to $2.7 \mathrm{~mm}$. The normalized $M_{0}$ recorded are presented in Fig. 1 as a function of optical path length. The maximum signal recorded is about a factor of 100 higher than the noise detected when the phase modulation is turned off. The full width at half maximum of the main peak is about $55 \mu \mathrm{m}$. With multimode interferometer, additional satellite peaks are observed at a distance of 60 and $300 \mu \mathrm{m}$ with respect to the main interference peak, which are at least a factor of 3.25 lower than the main peak. For widely different optical path lengths, the heterodyne signal is reduced to almost zero.

The deterioration of the path length resolution from $18 \mu \mathrm{m}$ (as expected from the coherence length of the light source) to about $55 \mu \mathrm{m}$ observed in both interferometers is due to the unbalanced optical dispersion of the reference and sample arms from the electro-optic modulator. ${ }^{9}$ The group dispersion of a $\mathrm{MgO}: \mathrm{LiNbO}_{3}$ crystal obtained from the slope of the curve of index of refraction of the crystal simulated with the Sellmeier equation $n^{2}(\lambda)=4.8762+\left[0.11554 /\left(\lambda^{2}\right.\right.$ $-0.04674)]-0.033119 \lambda^{2}$ ( $\lambda$ in micrometers) as a function of wavelength ${ }^{10}$ in the near-infrared spectral range $(815-842 \mathrm{~nm})$ is $D_{g}=-1.1 \times 10^{-4} / \mathrm{nm}$. When the reference beam is double passed through a crystal with a geometric length $L_{g}$, the reduced path length resolution is $L=\left[L_{c}^{2}\right.$ $\left.+\left(D_{g} L_{g} \Delta \lambda\right)^{2}\right]^{1 / 2}$. In our experimental configuration, the reference beam is passed through the crystal $\left(L_{g}=20 \mathrm{~mm}\right)$ only once and hence the reduced path length resolution is about $42 \mu \mathrm{m}$, which is in agreement with the path length resolution measured in our experimental setup. Path length resolution of $42 \mu \mathrm{m}$ is sufficient for measuring path length distributions of multiply scattered light, which often have widths of $5 \mathrm{~mm}$

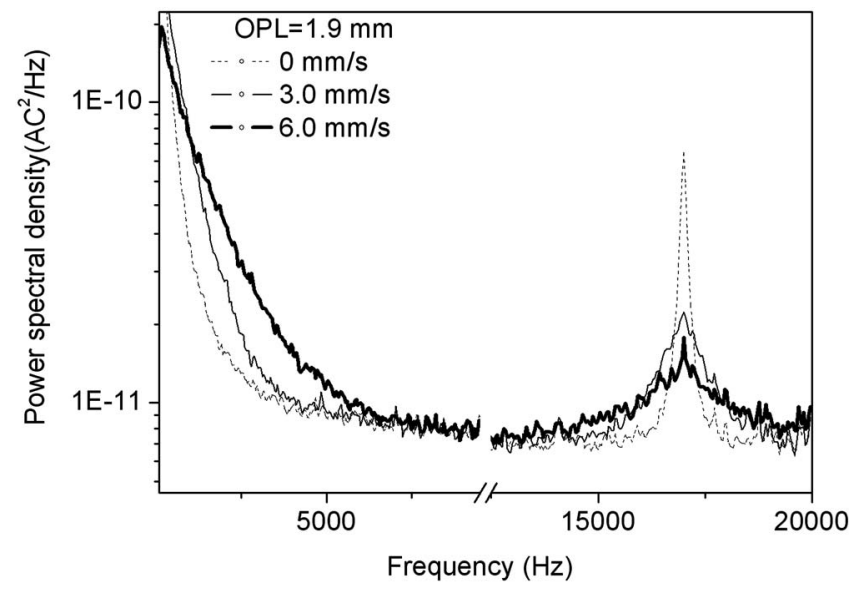

FIG. 2. Power spectral density of the detector current measured for an aqueous suspension of $10 \%$ of Intralipid $20 \%$ for different flow velocities, for an optical path length of $1.9 \mathrm{~mm}$.

or larger. Higher path length resolution may be obtained by compensating for the optical dispersion in the electro-optic modulator crystal by the introduction of an element with same amount of dispersion in the spectral range of the light source in the sample arm. ${ }^{11}$

The above results imply that the use of multimode graded index fibers does not cause serious degradation in the path length resolution compared to single mode fibers. However, additional satellite peaks are observed due to the intermodal dispersion in the graded index fiber. The upper limit of multimode dispersion of this fiber is specified as $1 \mathrm{~mm} / \mathrm{m}$. This value refers to the maximum difference in optical path length between lowest and highest fiber modes and it depends on how higher order modes are excited. The observed distance of the satellite peaks to the main peak is smaller than expected on the basis of the fiber specifications. In our path length resolution measurements with the mirror, probably a much smaller number of modes have been excited. The path length resolution of $100 \mu \mathrm{m}$ that we used in our measurements may result in suppression of other inherent modes. When measured in a scattering medium, higher order modes will be excited in a large core diameter fiber and the output intensity profile measured in the far field of the exit beam is the result of the superposition of all guided modes, resulting in a speckle pattern. The appearance of satellite peaks may lead to oscillations in the optical path length distributions at locations with strong second and higher order derivatives in the optical path length distribution. Since these peaks are very close to the main peak and the path length resolution is $100 \mu \mathrm{m}$, this will not be pronounced in the scattering media. The optical path length distributions measured in scattering media with different reduced scattering coefficients and anisotropies did not exhibit the effects of these satellite peaks. ${ }^{12}$ Furthermore, the choice of multimode fibers makes it possible to detect multiply scattered light at large fiber distances.

The broadening of the heterodyne peak observed in the power spectrum results from the Doppler shift imparted to the interfering light by the Brownian and translational motion of particles (Fig. 2). The spectrum of the interference signal becomes broad as the flow velocity increases. Since the optical properties of the medium are not changed due to 


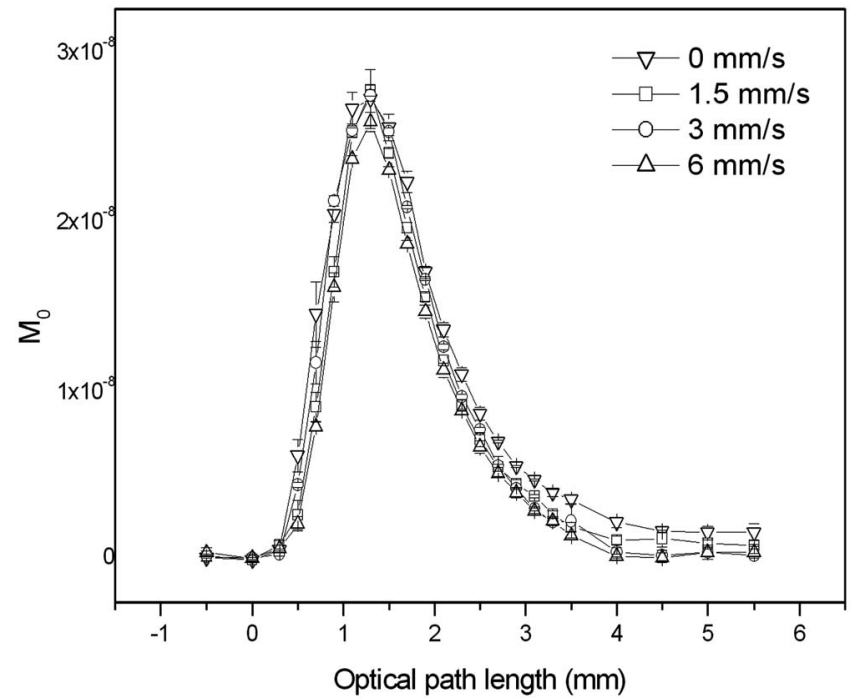

FIG. 3. Optical path length distributions measured for different flow velocities.

the flow, the area of the peak that corresponds to the intensity of photons within a certain optical path length should remain constant (Fig. 3). The error bars indicate the standard deviation of three measurements. The weighted first moment, which represents the average Doppler shift, increases with the optical path length due to multiple scattering (Fig. 4). For zero flow velocity, the average Doppler shift is not equal to zero due to the Brownian motion of the scattering particles. For a given optical path length, an increase in the average Doppler shift is observed when the diffusing particles experience translational motion. Since the peak may get drowned into the background noise for higher velocities, this may limit the usefulness of our technique for large flow velocities, for instance, blood flow measurements in arterioles $(6 \mathrm{~mm} / \mathrm{s}) .{ }^{13}$ Secondly, since majority of the photons will be

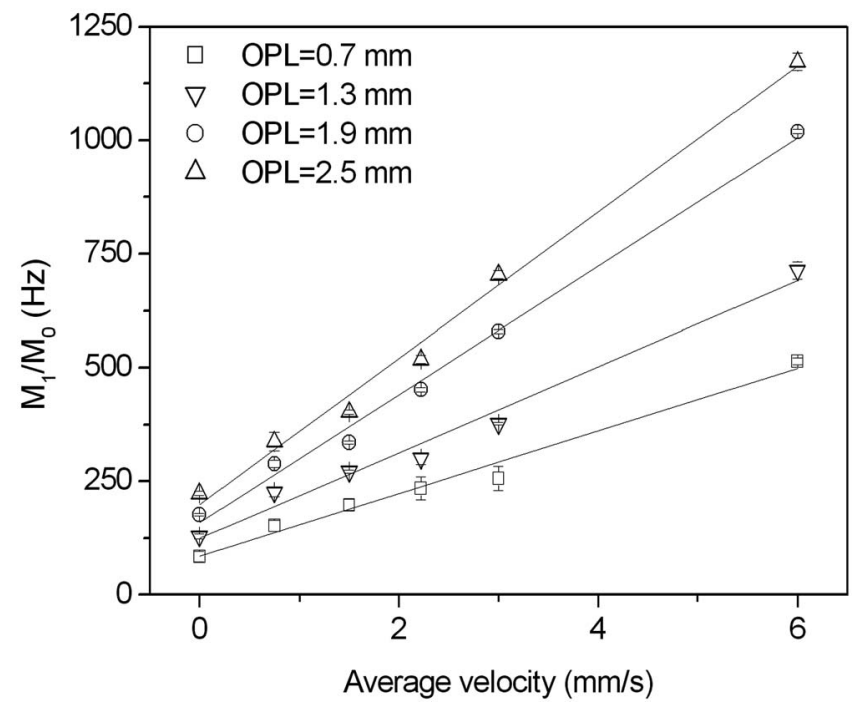

FIG. 4. The average Doppler shift measured as a function of optical path length for different velocities ranging from 0 to $6 \mathrm{~mm} / \mathrm{s}$. scattered by the surrounding static tissue matrices, less than $10 \%$ of the photons are associated with a Doppler shift generated by moving red blood cells. In the skin capillary network, the mean flow velocities of red blood cells through the small blood vessels are relatively lower, often in the range of $1 \mathrm{~mm} / \mathrm{s}^{13}$ In our measurements, for short optical path lengths, significant part of the photons will have traveled only within the static Delrin layer of thickness $0.2 \mathrm{~mm}$, and are thus not Doppler shifted, similar to the case of static tissue with small blood vessels. The Doppler shift estimated for a range of velocities from 0 to $6 \mathrm{~mm} / \mathrm{s}$ shows a linear response. This is an indication of the practical usability of our method in skin perfusion studies.

To summarize, in this paper, we presented path length resolved Doppler measurements of diffusely scattered light in an aqueous suspension of lipid particles undergoing Brownian and translational motion, using a phase modulated low coherence Mach-Zehnder interferometer with graded index multimode fiber for detection. The performance studies indicate that there is no significant effect of intermodal dispersion in the path length resolution of our experimental setup. The path length distributions measured in scattering media suggest that the effect of intermodal dispersion is very weak and the effect of additional satellite peaks that may result from the intermodal dispersion are not evident. The average Doppler shift increases with the optical path length, due to multiple scattering and shows a linear response with velocity for different flow velocities in the physiological range $(1 \mathrm{~mm} / \mathrm{s}$ in capillaries to $6 \mathrm{~mm} / \mathrm{s}$ in arterioles). Thus, multimode graded index fibers provide a large detection window with a small modal dispersion in path length resolved dynamic light scattering.

This work was sponsored by the Netherlands Technology Foundation STW (Grant No. TTF 5840) and the Institute for Biomedical Technology of the University of Twente.

${ }^{1}$ K. K. Bizheva, A. M. Siegel, and D. A. Boas, Phys. Rev. E 58, 7664-7667 (1998).

${ }^{2}$ A. Wax, C. Yang, R. R. Dasari, and M. S. Feld, Appl. Opt. 40, 4222 (2001).

${ }^{3}$ D. Huang, E. A. Swanson, C. P. Lin, J. S. Schuman, W. G. Stinson, W. Chang, M. R. Hee, T. Flotte, K. Gregory, C. A. Puliafito, and J. G. Fujimoto, Science 254, 1178 (1991).

${ }^{4}$ A. L. Petoukhova, W. Steenbergen, and F. F. M. de Mul, Opt. Lett. 26, 1492 (2001).

${ }^{5}$ A. L. Petoukhova, W. Steenbergen, T. G. van Leeuwen, and F. F. M. de Mul, Appl. Phys. Lett. 81, 595 (2002).

${ }^{6}$ B. Varghese, V. Rajan, T. G. Van Leeuwen, and W. Steenbergen, J. Biomed. Opt. 12, 024020 (2007).

${ }^{7}$ A. P. Shepherd and P. Å. Öberg, Laser-Doppler Blood Flowmetry (Kluwer/Academic, Boston, 1990).

${ }^{8}$ G. E. Nilsson, Med. Biol. Eng. Comput. 22, 343 (1984).

${ }^{9}$ C. K. Hitzenberger, A. Baumgartner, and A. F. Fercher, Opt. Commun. 154, 179 (1998).

${ }^{10}$ Sellmeier equations for $\mathrm{MgO}: \mathrm{LiNbO} 3$ crystal, Castech-Phoenix, Inc. (Casix), Fuzhou, Fujian 350014, China.

${ }^{11}$ X. C. Yao, A. Yamauchi, B. Perry, and J. S. George, Appl. Opt. 44, 2019 (2005).

${ }^{12}$ B. Varghese, V. Rajan, T. G. Van Leeuwen, and W. Steenbergen, Opt. Express 15, 9157-9165 (2007).

${ }^{13}$ A. C. Guyton, Textbook of Medical Physiology (Saunders, Philadelphia, 1991). 